\title{
Um Método de Modelagem de Processos para a Investigação de Problemas em Sistemas de Informação: Aplicação em uma Central de Regulação Médica
}

\author{
Alan P. Souza, João V. V. B. Freitas, Amauri M. Cunha, Maria L. M. Campos \\ Programa de Pós-Graduação em Informática (PPGI-IM/NCE) - Universidade Federal do \\ Rio de Janeiro (UFRJ) \\ Caixa Postal 2324 CEP: 20.001-970 - Rio de Janeiro - RJ - Brasil \\ \{alanpsouza, jvfreitas.freitas\}@gmail.com, \\ \{amauri, mluiza\}@nce.ufrj.br
}

\begin{abstract}
Emergency services for pre-hospital attendance are designed to provide quick mechanisms for medical care. These services, in general, have some kind of computational system to support the execution of its activities and to register realized tasks. This paper presents a method that uses process modeling for identifying problems in an information system used in a medical regulation center of a large city. The results obtained in implementing the method show its applicability.
\end{abstract}

Resumo. Os serviços de emergência para atendimento pré-hospitalar têm como objetivo prover mecanismos ágeis de assistência médica. Estes serviços, em geral, possuem algum tipo de sistema computacional para suporte à execução das suas atividades e ao registro dos atendimentos efetuados. Este artigo apresenta um método que utiliza a modelagem de processos para a identificação de problemas existentes no sistema de informação utilizado em uma central de regulação médica de uma metrópole nacional. Os resultados obtidos com a execução do método mostram a sua aplicabilidade.

\section{Introdução}

Levantamentos e pesquisas diversas apontam que muitos dos óbitos associados a acidentes ou ocorrências de emergência médica poderiam ser evitados se houvesse algum atendimento médico logo nos primeiros instantes após a ocorrência do evento [Elliot 2000]. O tempo de atendimento a uma vítima é um fator crítico para a sobrevivência, especialmente em situações traumáticas. Por esta razão, justifica-se a busca por mecanismos que acelerem o processo de atendimento. Para atender a estas necessidades, diversos serviços de atendimento pré-hospitalar já foram criados há algum tempo, sendo alguns destes modelos reconhecidos mundialmente ${ }^{1}$. No Brasil, estes serviços para o atendimento a eventos de urgência e emergência foram instituídos mais

\footnotetext{
1 Alguns destes serviços existem há décadas, com destaque para aqueles adotados na França (Service d'Aide Medicale d'Urgence) e nos Estados Unidos (Emergency Medical Services Systems Act).
} 
recentemente $^{2}$, quando o Ministério da Saúde decidiu implantar um componente préhospitalar móvel em municípios e regiões de todo o país [Ministério da Saúde 2006a].

Este trabalho é fruto de um projeto que tem como objetivo propiciar melhorias aos atendimentos oferecidos por um serviço pré-hospitalar de uma metrópole nacional, através do desenvolvimento e implantação de soluções baseadas em tecnologias de informação.

A abrangência deste projeto vai desde o tratamento das solicitações telefônicas, a fim de melhorar a produtividade operacional do sistema, passando pelo estudo da cognição humana nos postos de trabalho em situações de urgência, até ao processo de gerenciamento dinâmico das ambulâncias e suas equipes de atendimento associadas. Entretanto, o escopo deste trabalho se concentra em minorar a dificuldade de processamento das chamadas telefônicas, devido às possíveis problemas do sistema computacional de regulação médica implantado na central de atendimento, tendo como meta a redução no tempo de registro das informações provenientes de uma ocorrência no software de apoio às atividades do serviço pré-hospitalar.

Existem descontentamentos naqueles que operam o sistema computacional de apoio à central de atendimento, havendo muitas reclamações de que o mesmo provoca atrasos no tratamento das solicitações telefônicas no atendimento de urgências médicas.

Com o objetivo de identificar causas para os problemas apresentados, a equipe de projeto realizou um trabalho de campo para levantar o processo de trabalho dos operadores da central de regulação médica, incluindo a avaliação dos sistemas informacionais utilizados. O estudo exploratório de campo através de entrevistas com pessoas específicas envolvidas no serviço utilizou como base a metodologia preconizada por Thiollent (2003).

Seguindo as propostas de Cunha e Souza $(2005,2006)$, através da utilização do conceito de memória, buscou-se rastrear os recursos utilizados, de modo a adquirir uma ampla compreensão das atividades executadas. Com a análise dessas atividades esperase identificar problemas na concepção e utilização do sistema computacional, assim como outros oriundos de procedimentos inadequados de trabalho humano.

Este tipo de trabalho é voltado para o levantamento detalhado de processos envolvendo as pessoas que operam a central de regulação médica, utilizando a suposição largamente aceita de que esta é uma forma adequada de estudar os sistemas informacionais de apoio ao trabalho humano [Sharp e McDermott 2001].

As sucessivas visitas à central de regulação deste serviço de atendimento préhospitalar foram relevantes em razão da possibilidade de lidar com uma variedade de evidências, visto estar o serviço de emergência inserido dentro de um contexto amplo e complexo, que inviabilizava sua compreensão fora da conjuntura na qual ocorre.

\footnotetext{
${ }^{2}$ Os serviços de atendimento pré-hospitalar foram definidos através da portaria $\mathrm{n}^{\circ} 1.864$, de 29 de setembro de 2003 .
} 
Outro aspecto interessante é que esses encontros permitiram o estabelecimento de relações de confiança com os diferentes profissionais envolvidos no serviço ${ }^{3}$, o que foi fundamental para maior aceitação no desenvolvimento do estudo investigativo. Apesar do ritmo das atividades na central de regulação ser intenso e desgastante para cada um dos profissionais envolvidos, essas características pouco afetaram o andamento do estudo, pois a maior parte dos levantamentos foi realizada junto aos chefes de operação e equipes de apoio; os profissionais diretamente envolvidos no atendimento das ocorrências foram pouco exigidos.

Para evitar o ceticismo por parte dos indivíduos que foram alvo da pesquisa com relação às teorias, métodos e outros elementos valorizados pelo espírito científico [Thiollent 2003], a cada passo dado no desenvolvimento do estudo, os profissionais do serviço foram sendo apresentados, de maneira gradual, às diversas técnicas que estavam sendo utilizadas.

Este artigo inclui além desta introdução outras cinco seções descritas a seguir. Na seção 2 apresenta-se o contexto dos serviços de atendimento pré-hospitalar. A seção 3 discute o referencial teórico, evidenciando como a modelagem de processos pode ser favorável no levantamento de requisitos dos sistemas de informação. Na seção 4, o método utilizado é detalhado, assim como os resultados obtidos a partir de sua aplicação. Finalmente, apresentam-se as conclusões na seção 5.

\section{Serviço de Atendimento Pré-Hospitalar (APH)}

$\mathrm{O}$ atendimento pré-hospitalar compreende qualquer assistência médica realizada fora do âmbito hospitalar, provendo assistência e transporte adequado até algum serviço de saúde, devendo este procedimento ser efetuado somente por profissionais especializados [Junior e Valente 2005].

Lopes e Fernandes (1999) consideram APH como toda assistência realizada, de forma direta ou não, fora do âmbito hospitalar, através dos diversos meios e métodos disponíveis, com uma resposta adequada à solicitação de socorro, tendo como objetivo a manutenção da vida e/ou a minimização das seqüelas.

Segundo o Conselho Federal de Medicina [apud Ministério da Saúde 2006b], uma Emergência é a constatação médica de condições de agravo à saúde que impliquem em risco iminente de vida ou sofrimento intenso exigindo, portanto, tratamento médico imediato. Urgência é a ocorrência imprevista de agravo à saúde com ou sem risco potencial de vida, cujo portador necessita de assistência médica imediata.

Este serviço envolve uma equipe de profissionais de saúde preparada para atender ao paciente da melhor maneira possível, contando também com o suporte especializado de tele-atendentes, operadores de frota, condutores de ambulância, etc. A Figura 1 apresenta o processo relacionado ao serviço de atendimento pré-hospitalar.

\footnotetext{
${ }^{3}$ Durante as visitas realizadas todos os membros da equipe do projeto estabeleceram diálogos com os mais diferentes papéis associados ao serviço: coordenadores, administradores, auxiliares técnicos, supervisores, tele-atendentes, médicos reguladores, operadores de frota, etc.
} 


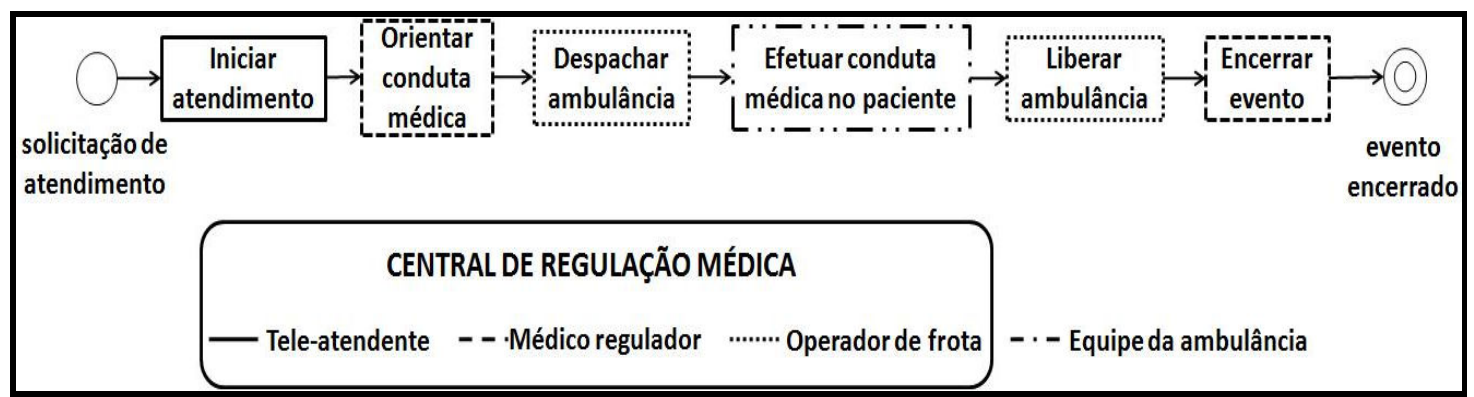

Figura 1 - Fluxo de atividades simplificado relativo ao atendimento de uma ocorrência.

O processo tem início com o recebimento de uma solicitação de socorro por telefone. Neste momento, uma tele-atendente realiza de maneira concomitante o registro de dados do evento e a triagem de possíveis ligações inadequadas (trotes, enganos, etc.).

Sendo a ligação definida como apropriada, a mesma é transferida para um médico regulador responsável por realizar a avaliação do cenário clínico. Nesta etapa, o médico vai realizar o diagnóstico do paciente, decidindo pelo envio ou não de uma ambulância para o local da ocorrência [Borsato et al 2006].

O médico regulador pode dar um conselho, uma orientação, ou deslocar uma equipe com médico e enfermeiro e todos os equipamentos de uma UTI. A liberação de cada recurso será específica para a necessidade de cada ocorrência. Em caso positivo, um operador de frota é incumbido da alocação e despacho de uma ambulância específica (a depender da gravidade da ocorrência).

A equipe de socorro enviada para o local da ocorrência vai submeter o paciente a condutas médicas, onde pode ser identificada ou não a necessidade de se transferir o paciente para uma unidade de saúde.

Ao término do atendimento, a equipe da ambulância deve comunicar ao operador de frota a conclusão do mesmo. Este profissional deve liberar a ambulância associada ao atendimento, para que o médico regulador possa definir a ocorrência como encerrada.

\section{Modelagem de Processos para Elicitação de Requisitos}

Os requisitos de um software são definidos como as propriedades que um sistema deve possuir para satisfazer as necessidades do ambiente em que será utilizado. Apesar da importância de existir um processo de elicitação de requisitos de qualidade, a maior parte dos sistemas é desenvolvida sem uma perfeita compreensão das necessidades do negócio, de modo que é possível vislumbrar um panorama onde a qualidade de software nos dias atuais é muito ruim [Pressman 2006].

$\mathrm{Na}$ literatura não foram encontrados trabalhos relevantes que abordam a utilização da modelagem de processos para a identificação de problemas em sistemas computacionais. A maioria dos estudos envolve a utilização da modelagem no levantamento de requisitos para a elaboração de casos de uso [Jacobson, Ericsson e Jacobson 1994; Sharp e McDermott 2001].

Dentro do contexto deste projeto de pesquisa, a modelagem de processos foi a metodologia utilizada tanto para a equipe de pesquisadores absorver conhecimentos sobre os procedimentos adotados na central de regulação médica, como forma de 
identificar um conjunto de problemas existentes no sistema utilizado, assim como os requisitos realmente relevantes que o sistema de informação deve possuir para atender as suas exigências.

Aliada à construção dos modelos de processos, outra técnica bastante valiosa é o destaque dos recursos que são manipulados ao longo do fluxo de atividades, identificando os insumos necessários para a execução de uma determinada atividade e os resultados provenientes do cumprimento desta. A relevância desses elementos informacionais, identificados como memórias, é destacada no trabalho de Cunha e Souza (2006) e visualizados neste artigo através da Figura 2.

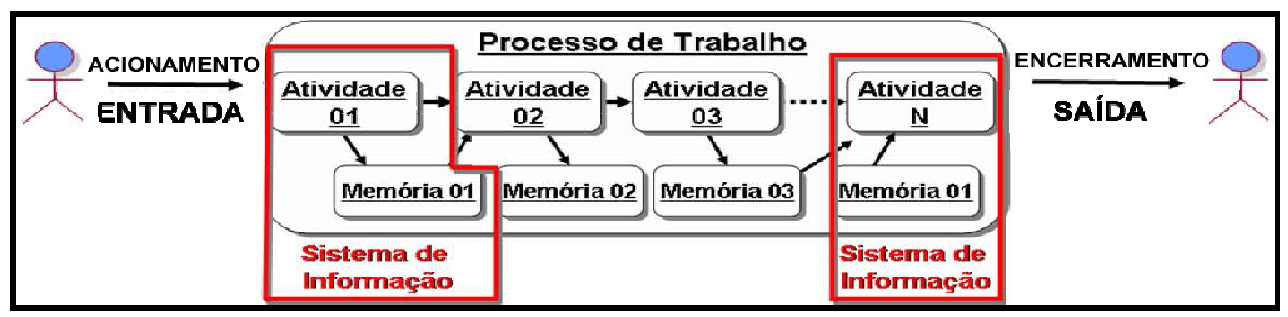

Figura 2 - Ênfase do conceito de memórias no modelo de processos de negócios identificando as atividades que terão apoio de TI, adaptado de [Cunha e Souza 2006].

Uma memória representa qualquer informação gerada e armazenada em um meio persistente ${ }^{4}$ da organização para ser consultada ou atualizada posteriormente com algum objetivo pelas atividades do processo [Cunha e Souza 2006].

Outra vantagem da utilização de memórias é a possibilidade de reconhecer informações desnecessárias. Isto ocorre quando a memória é criada por uma atividade e não é utilizada por nenhuma outra atividade dos processos existentes.

O RUP (Rational Unified Process) apresenta os artefatos em sua estrutura, que são os elementos que mais se assemelham ao conceito de memória. Nesta metodologia, os artefatos representam produtos no processo, gerados ou consumidos durante a execução de uma atividade. $\mathrm{O}$ seu ponto negativo é o fato de desconsiderar como artefato os elementos informacionais que não estejam armazenados em algum tipo de relatório. Assim, comunicações verbais relevantes para o processo podem não ser levadas em conta [Rational Unified Process 2000].

Com base neste referencial teórico a equipe de projeto promoveu a modelagem de processos, rastreando as informações utilizadas ao longo do fluxo de atividades, e identificando os recursos computacionais e humanos responsáveis pelo seu manuseio. A proposta desenvolvida neste projeto é descrita na próxima seção, onde o método utilizado é apresentado com mais detalhes.

\section{Método Adotado}

Esta seção apresenta o levantamento realizado para identificar os problemas existentes nas atividades executadas dentro da central de regulação médica. Como evidência desses problemas, está o não cumprimento do tempo máximo desejado para um serviço de atendimento de urgência [Elliot 2000]. O principal desafio foi identificar os

\footnotetext{
${ }^{4}$ Esses meios persistentes podem ser os bancos de dados da organização, arquivos em formato texto ou planilhas, além de documentos em papel, livros de anotações, etc.
} 
problemas existentes no sistema computacional utilizado, e para isso foi utilizado o seguinte método:

\section{Coleta de Dados}

i. Estudo da documentação existente, tendo como objetivo o entendimento do domínio de conhecimento da organização.

ii. Observação in loco da execução do processo de trabalho.

iii. Entrevistas com profissionais chaves para o processo de trabalho.

2. Análise de Dados

i. Compreensão detalhada do Processo de Trabalho

a. Papéis associados à execução das atividades do processo de trabalho.

b. Insumos e produtos relacionados às atividades.

c. Suporte computacional de apoio à execução das atividades.

d. Recursos utilizados como apoio à execução do processo (Formulários suplementares, planilhas em meio computacional, etc.)

ii. Elaboração do Glossário de Termos

iii. Análise inicial dos Problemas relacionados ao Suporte Computacional

a. Atividades que não possuem suporte computacional.

b. Atividades que possuem suporte computacional, mas com dependência de recursos suplementares.

3. Estruturação do conhecimento a respeito dos Processos de Trabalho

i. Modelagem do processo de trabalho

a. Eventos associados ao início e término do processo, além de eventos temporais inicializados durante a execução do mesmo.

b. Papéis responsáveis pela execução das atividades do processo de trabalho.

c. Atividades do processo de trabalho com seus respectivos insumos e resultados.

I. Incluindo atividades que representem repetições.

d. Pontos de decisão presentes no processo de trabalho.

ii. Revisão do Glossário de Termos

4. Modelagem Conceitual dos Dados baseada nas Memórias Organizacionais.

i. Especificação das memórias organizacionais presentes no processo de trabalho, independentemente do meio que estejam persistidas.

ii. Análise do relacionamento existente entre as memórias organizacionais identificadas no processo de trabalho.

iii. Revisão do Modelo Conceitual de Dados a partir das Memórias Organizacionais especificadas e seus relacionamentos.

5. Repetir os passos de $\mathbf{1}$ a $\mathbf{4}$ para cada um dos processos de trabalho identificados

6. Estudo dos Problemas relacionados ao Suporte Computacional nas atividades de cada um dos processos de trabalho

i. Atividades que consomem ou produzem memórias organizacionais, mas não possuem suporte computacional.

ii. Atividades que possuem suporte computacional, mas as memórias organizacionais consumidas ou produzidas não são contempladas.

iii. Atividades com suporte computacional inadequado, onde as memórias organizacionais não são contempladas de forma apropriada.

\subsection{Detalhamento}

Como resultados das atividades de campo foram gerados diferentes tipos de documentos para cobrir necessidades de diferentes etapas do projeto ${ }^{5}$. Dessa forma, a

\footnotetext{
${ }^{5}$ Em suas diferentes etapas, o método aplicado gerou diversos tipos de documentos, entre eles: diagramas de processos com insumos e resultados das atividades, mapas conceituais, glossários de termos, dicionários e modelos de dados, etc.
} 
equipe de trabalho ficou mais capacitada para formular hipóteses de melhorias ou ao menos fazer progredir a consciência quanto à existência de obstáculos para $o$ funcionamento satisfatório do serviço.

Inicialmente foram realizados encontros da equipe do projeto com coordenadores e profissionais envolvidos nas atividades do serviço, tendo como objetivo a aquisição de um conhecimento básico sobre o fluxo de atividades do processo completo de regulação médica, nos próprios locais onde as atividades são executadas.

A partir daí, de maneira gradual, através da análise das entrevistas gravadas, do estudo das apostilas de treinamento do sistema computacional e da avaliação dos formulários manuais preenchidos, os pesquisadores passaram a elaborar vários modelos de processos, juntamente com novas versões de todos os documentos utilizados.

A cada semana, novas reuniões eram agendadas na central de regulação com os profissionais apropriados de modo que estes pudessem validar as documentações produzidas. As discussões que surgiam agregavam sempre novos conhecimentos, correções e sugestões para os documentos e relatórios do projeto.

$\mathrm{Na}$ modelagem de processo, os papéis atuantes em cada uma das atividades foram discriminados visualmente através do uso de piscinas e raias, além disso, para cada uma das atividades, os insumos e resultados ${ }^{6}$ associados foram evidenciados através do conceito de memória [Cunha e Souza 2006]. Os diagramas de processos construídos ressaltavam ainda, através de códigos identificadores, em qual tipo de meios persistentes essas memórias se encontravam (por exemplo, se em sistema computacional, formulário em papel, etc.).

A comunicação entre os profissionais, as decisões, os processos temporais, enfim, todos os aspectos que foram classificados como relevantes para a futura identificação dos problemas e as suas causas foram de alguma forma registrados nos modelos. A Figura 3 mostra uma pequena parte de um dos modelos de processos, evidenciando estes aspectos.

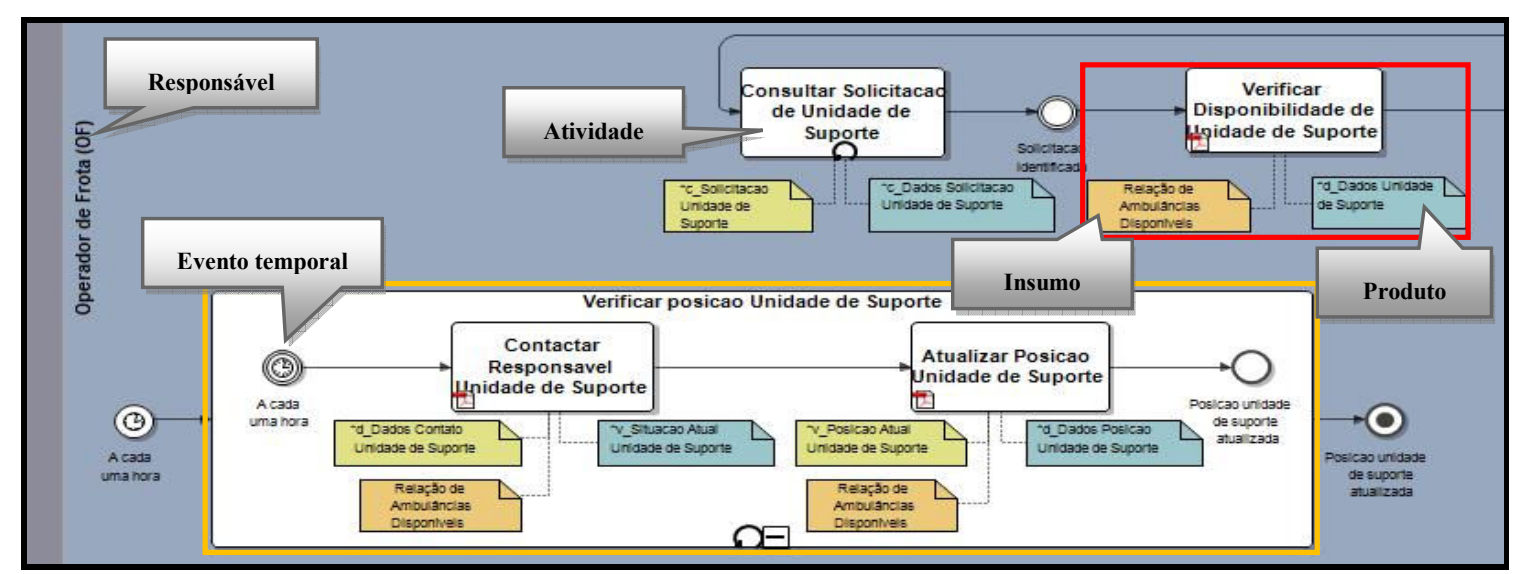

Figura 3 - Parte de um modelo do processo de atendimento do serviço de atendimento préhospitalar adotado na Central de Regulação Médica.

\footnotetext{
${ }^{6}$ Insumo é tudo aquilo que uma atividade precisa obter de seu exterior para ser completamente realizada e gerar suas saídas. Resultado é tudo aquilo que a atividade emite para o seu exterior durante a sua execução [Cunha e Souza 2006].
} 
Em paralelo, foram gerados mapas conceituais ${ }^{7}$ e glossários de informações refletindo aquilo que era representado nos modelos, visando representar o conhecimento obtido. Toda a documentação elaborada passou por reuniões de aprovação até se atingir um detalhamento definido como satisfatório para as atividades do projeto.

\subsection{Resultados Obtidos}

Os resultados obtidos neste estudo estão baseados nas orientações contidas nos modelos de processos de trabalho do domínio de atendimento pré-hospitalar e transferência interhospitalar, detalhados através da proposta de caracterização de atividades definida em Cunha e Souza (2006), o que possibilitou a representação de todos os insumos e produtos dos mesmos, sendo estes informacionais, materiais ou verbais.

Através deste detalhamento foi possível iniciar uma análise das inadequações do sistema de informação e dos problemas de utilização do mesmo, listados a seguir:

\section{O emprego de recursos suplementares}

Um dos primeiros resultados obtidos através desta análise foi à identificação do uso excessivo de papel em atividades já suportadas pelo sistema de informação, como a utilização em paralelo de formulários em papel na persistência de informações necessárias à execução do processo, provocando uma redundância de dados.

Este fato ocorre devido à desconfiança quanto ao desempenho do sistema de informação, principalmente em relação aos seus requisitos não funcionais de confiabilidade e desempenho [Sommerville 2003]. Esta prevenção é justificada pelas constantes interrupções e falhas apresentadas pelo sistema, tendo como conseqüência um maior esforço das pessoas e maior utilização de recursos materiais durante a execução das atividades.

A utilização destes formulários resultou no desenvolvimento de base de dados que tentam "espelhar" a estrutura de dados contida nos mesmos, sendo povoadas a posteriori por uma equipe de digitadores. A necessidade deste registro subseqüente representa um acréscimo nos custos associados à execução do processo, além de trazer problemas como a replicação e inconsistência dos dados registrados nestas diversas bases, já que elas não são integradas e possuem diferentes representações dos conceitos do domínio da aplicação.

\section{A identificação de atividades não suportadas pelo sistema de informação}

A modelagem do processo de trabalho possibilitou o reconhecimento de atividades não apoiadas pelo sistema de informação. Um exemplo desta situação é o controle realizado de forma manual pelos operadores de frota $(\mathrm{OF})$ em relação à disponibilidade das ambulâncias e à disposição das mesmas nas bases geográficas de estacionamento, devido à inexistência dessas funcionalidades no sistema de informação.

Como forma de suprir essa deficiência, outro conjunto complementar de documentos em papel foi elaborado e passou a ser utilizado, tendo como objetivo registrar o conjunto de ambulâncias disponíveis. Esses formulários são atualizados

\footnotetext{
7 Mapa conceitual é um diagrama (ou grafo direcionado) que possui nós e associações. Os nós representam conceitos do domínio, onde cada conceito pode ser expresso por uma palavra, algumas palavras ou até mesmo símbolos. Os conceitos são relacionados através das associações.
} 
diariamente por recursos especialmente alocados para tal fim, tendo como resultado um maior esforço na execução destas atividades e insegurança quanto à utilização dos dados, uma vez que eles são disponibilizados em papel, com dificuldades para manutenção.

\section{Inadequações do sistema de informação}

O levantamento dos processos de trabalho também possibilitou a identificação de atividades onde as funcionalidades do sistema de informação representavam algum tipo de "gargalo" para a execução do processo. Esta situação foi percebida quando da necessidade de se encerrar um atendimento realizado com o auxílio de uma ambulância.

Idealmente, o responsável pela comunicação na ambulância deveria informar ao operador de frota sobre a conclusão do atendimento, permitindo ao mesmo liberar a ambulância no sistema, retornando-a para a lista de ambulâncias livres, e comunicar ao médico regulador responsável a possibilidade de encerrar o atendimento no sistema de informação. Contudo, o médico regulador não possui permissão para encerrar um atendimento que ainda possua uma ambulância associada e se vê impossibilitado de proceder tal tarefa enquanto o término das comunicações entre a ambulância e $o$ operador de frota não forem formalizadas no sistema computacional.

Além disso, devido ao fato de o sistema de informação não oferecer uma forma automática de comunicação quando da liberação das ambulâncias, o número de atendimentos aguardando fechamento, mas já terminados na prática, passam a representar boa parte dos atendimentos em execução, trazendo sobrecarga ao funcionamento do sistema de informação. Esta situação contribui para agravar as suas constantes interrupções e falhas.

\section{Análise de Modelo de Dados}

Outro resultado obtido com a aplicação da proposta de processo de trabalho está relacionado à análise do modelo conceitual de dados. Como citado anteriormente, informações refletindo aquilo que era representado nos processos de trabalho foram documentadas em glossários e mapas conceituais, de modo a gerar relatórios de suporte para uma maior compreensão do conhecimento sendo elaborado.

Todos os termos representados nos modelos de processos de trabalhos e documentados no glossário e mapas conceituais apresentam descrições bastante ricas. Desta forma, a partir destes elementos, foi possível desenvolver modelos conceituais de dados que detalham o conjunto de dados pertencentes ao domínio, assim como os seus relacionamentos. As memórias utilizadas em várias atividades, independente do meio em que eram persistidas ou por quais papéis eram manipuladas, representam conceitos de maior importância dentro do domínio relacionado ao processo de trabalho. Desta forma, estes elementos informacionais devem ser mapeados como uma entidade do modelo conceitual de dados, pois estes estão ou pelo menos deveriam estar associados a alguma funcionalidade do sistema computacional que suporta o processo de trabalho.

Sendo assim, o estudo integrado desses modelos permite uma análise detalhada dos requisitos do sistema, auxiliando o reconhecimento das funcionalidades relevantes, inadequadas ou desnecessárias. A Figura 4 apresenta um exemplo do mapeamento de uma memória identificada no processo de trabalho, com sua definição especificada no glossário de termos do domínio, para uma entidade do modelo conceitual de dados. 


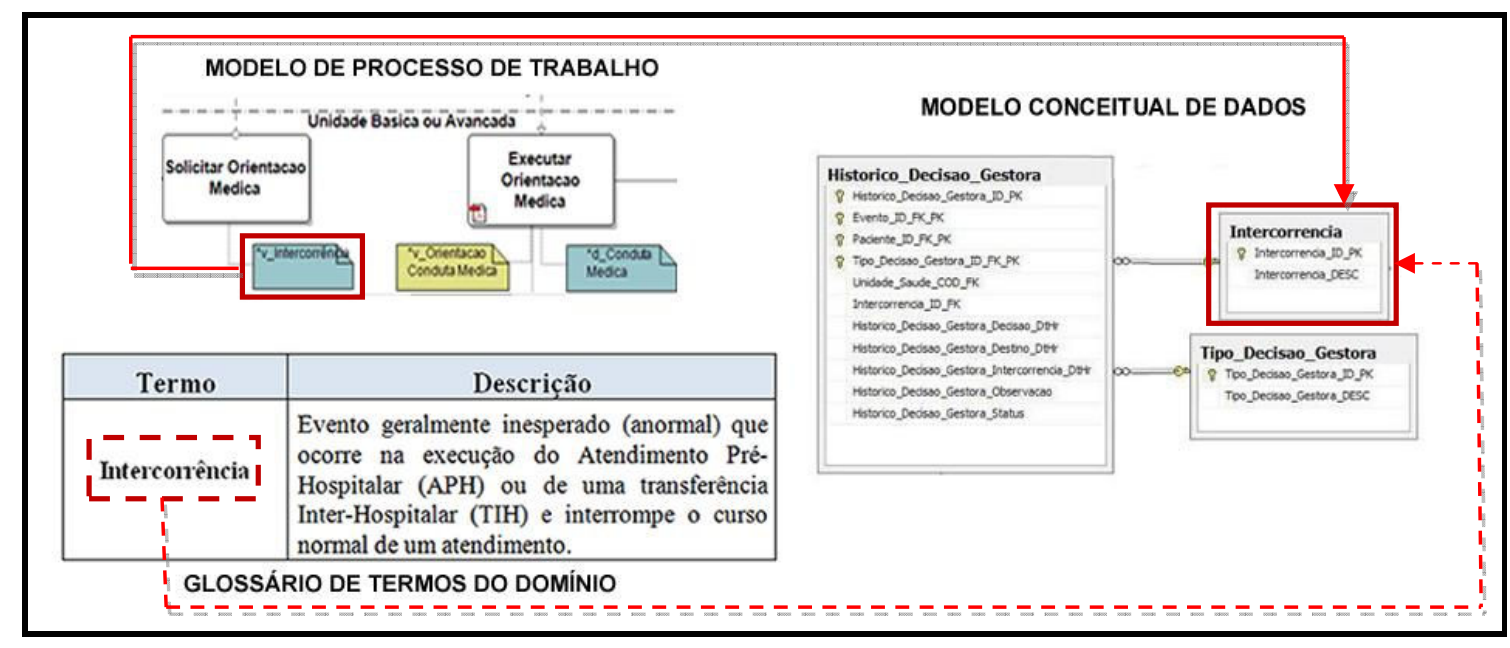

Figura 4 - Mapeamento entre os diferentes dispositivos de tratamento de dados.

A partir destes modelos de dados, segue a construção de um Operational Data Store $(O D S)$, com a eliminação das devidas inconsistências, através da realização dos procedimentos de extração, carga e transformação dos dados. Os primeiros data marts possibilitarão o desenvolvimento de ambientes amigáveis de visualização dos dados e geração flexível dos relatórios gerenciais.

\section{Conclusões}

A identificação de inadequações e problemas de utilização de um sistema de informação não é uma atividade trivial, pois envolve a análise de aspectos técnicos, humanos e organizações, além da relação entre os mesmos. No domínio relacionado ao atendimento pré-hospitalar esta análise torna-se ainda mais complexa, dado o stress envolvido na sua execução.

Neste sentido, foi possível observar que o método apresentado na seção 4 mostrou-se adequado na identificação de impropriedades e problemas de utilização do sistema de informação apontados na seção 4.2 deste artigo.

Outro aspecto a ser ressaltado foi a utilização do conceito de memória como forma de caracterização dos processos de trabalho e de suas respectivas atividades, pois através deste conceito foi possível identificar todos os elementos informacionais e materiais associados aos processos. Além disso, através do conceito de memória foi possível identificar atividades que apresentavam duplicação de informações, como o emprego de recursos suplementares, informações relacionadas ao domínio, mas não suportada pelo sistema de informação, assim como inadequações apresentadas pelo mesmo.

Além destes resultados de âmbito mais técnico, foi possível observar que os modelos de processos de trabalho podem apoiar a realização de treinamentos dos funcionários envolvidos nas atividades da central de regulação médica. A aquisição do conhecimento seria facilitada, pois os modelos de processo além de representarem de maneira correta como as atividades devem ser executadas, possuem ainda um forte aspecto visual, que confere destaque às responsabilidades que cada papel possui dentro do fluxo de atividades, assim como os recursos informacionais associados a cada atividade. 
O projeto encontra-se em andamento, estando na fase de análise de dados e definição de protótipos em laboratório, sendo que os modelos resultantes da aplicação do método estão servindo de base para ambas.

As próximas etapas do projeto incluem o desenvolvimento de um banco de dados integrado, baseado nos modelos de dados elaborados na etapa anterior, que possa receber dados de todos os registros hoje utilizados. Este banco será utilizado como alicerce de um ambiente de suporte a aplicações analíticas que permitirão a geração sistemática de relatórios gerenciais, capazes de prover periodicamente informações sobre os serviços prestados.

A expectativa é que este ambiente analítico auxilie a produção de estatísticas mais confiáveis em relação aos atendimentos existentes, especialmente aquelas associadas às medidas de duração de atividades, de modo a apoiar futuras tomadas de decisões.

Durante o trabalho realizado foram identificadas limitações como, por exemplo:

- Alto custo de aplicação do método, em razão das muitas interações para se gerar e validar sucessivas versões de documentos;

- Dificuldade de reunir as pessoas mais adequadas em cada um dos encontros realizados e a geração de extensos volumes de gravações em áudio;

- Profissionais da central de regulação médica gastaram um tempo considerável na familiarização e compreensão das técnicas e ferramentas adotadas;

- Falta de garantia se todas as impropriedades do sistema foram identificadas;

- Falta de um sistema computacional para apoiar as atividades do método utilizado.

E, por fim, algumas necessidades percebidas ou aspectos não contemplados por este trabalho surgem como sugestões para trabalhos futuros, entre eles: a análise detalhada de todas as interfaces do sistema de informação; a identificação de problemas oriundos de procedimentos de trabalho humano; o desenvolvimento de um ambiente integrado para suportar as etapas do método utilizado; e validação do método em outros tipos de sistemas.

\section{Referências Bibliográficas}

Borsato, G.B., Scalabrin E.E., Dias J.S. e Enembreck, F. (2006). "Sistema de Apoio à Tomada de Decisão para Atendimento Pré-Hospitalar”, In: CBIS'2006 - X Congresso Brasileiro de Informática em Saúde. Florianópolis.

Cunha, A. M. e Souza, G. Q. (2005). "Especificando Requisitos a Partir do Conceito de Processo de Trabalho", In: VII SIMPROS - Simpósio Internacional de Melhoria de Processo de Software, São Paulo.

Cunha, A. M. e Souza, G. Q. (2006). "O Conceito de Memória na Modelagem de Processos de Negócios para Gerar Requisitos de Sistemas de Informação", In: III SBSI - Simpósio Brasileiro de Sistemas de Informação, Curitiba. 
Destri, J. (2005). "Sistema de Apoio à Decisão Espacial Aplicado ao Serviço de Atendimento Móvel de Urgência em Vias de Trânsito", Universidade Federal de Santa Catarina, Florianópolis.

Elliot, P. (2000). “An Approach to Integrated Rescue”. QMC - Queensland Mining Council: Osborne Mines.

Goguen, J. (1992). “The Dry And The Wet In Information Systems Concepts: Improving The Understanding”, In: Proceedings IFIP WG 8.1 Conference, Elsevier Holland.

Jacobson. I., Ericsson, M and Jacobson, A. (1994). "The Object Advantage, Business Process Reengineering With Object Technology". Addison-Wesley Publishing Company, ACM Press.

Junior, J. e Valente, A. (2005). “A Logística no Serviço de Atendimento Móvel de Urgência (SAMU)", XXV Encontro Nacional de Engenharia de Produção, Porto Alegre.

Lopes, S. e Fernandes, R. (1999). "Uma Breve Revisão do Atendimento Médico PréHospitalar”, Medicina, Ribeirão Preto, 32: 381-387, outubro/dezembro.

Ministério da Saúde (2006a). "Política Nacional de Atenção às Urgências", Série E, Legislação de Saúde, Brasília.

Ministério da Saúde (2006b). "Regulação Médica das Urgências", Série A, Normas e Manuais Técnicos, Brasília.

Pressman, R. (2006). "Engenharia de Software”, Sexta Edição. McGraw-Hill.

Rational Unified Process (2000), Rational Software, Cupertino, Califórnia. Disponível em: http://www.rational.com/rup/. Acessado em: 16 de março de 2009.

Santos, A. (2005). "Modelagem de Processos de Negócios como Base para a Elicitação de Requisitos de Software", Universidade Federal da Bahia.

Sharp, A. and McDermott, P. (2001). "Workflow Modeling”, Artech House Inc.

Sommerville, I. (2003). "Engenharia de Software", Editora Pearson Addison Wesley, São Paulo.

Tani, V. (2003). "SAD baseado em Caminhos Mínimos e Georeferenciamento: uma Ferramenta de Apoio ao Serviço de Regulação Médica", Universidade Federal de Santa Catarina, Florianópolis.

Thiollent, M. (2003). "Metodologia da Pesquisa-Ação", Cortez Editora. 